\title{
Filifactor alocis - involvement in periodontal biofilms
}

\author{
Sebastian Schlafer', Birgit Riep², Ann L Griffen³ , Annett Petrich', Julia Hübner', Moritz Berning ${ }^{1}$, Anton Friedmann²,
} Ulf B Göbel ${ }^{1}$, Annette Moter ${ }^{1 *}$

\begin{abstract}
Background: Bacteria in periodontal pockets develop complex sessile communities that attach to the tooth surface. These highly dynamic microfloral environments challenge both clinicians and researchers alike. The exploration of structural organisation and bacterial interactions within these biofilms is critically important for a thorough understanding of periodontal disease. In recent years, Filifactor alocis, a fastidious, Gram-positive, obligately anaerobic rod was repeatedly identified in periodontal lesions using DNA-based methods. It has been suggested to be a marker for periodontal deterioration. The present study investigated the epidemiology of F. alocis in periodontal pockets and analysed the spatial arrangement and architectural role of the organism in in vivo grown subgingival biofilms.

Results: A species-specific oligonucleotide probe, FIAL, was designed and evaluated. A total of 490 subgingival plaque samples were submitted to PCR and subsequent dot blot hybridization to compare the prevalence of F. alocis in patients suffering from generalized aggressive periodontitis (GAP), chronic periodontitis (CP), and control subjects resistant to periodontitis. Moreover, a specially designed carrier system was used to collect in vivo grown subgingival biofilms from GAP patients. Subsequent topographic analysis was performed using fluorescence in situ hybridization.

While the majority of patients suffering from GAP or CP harboured F. alocis, it was rarely detected in the control group. In the examined carrier-borne biofilms the organism predominantly colonized apical parts of the pocket in close proximity to the soft tissues and was involved in numerous structures that constitute characteristic architectural features of subgingival periodontal biofilms.
\end{abstract}

Conclusions: $F$. alocis is likely to make a relevant contribution to the pathogenetic structure of biofilms accounting for periodontal inflammation and can be considered an excellent marker organism for periodontal disease.

\section{Background}

Periodontitis is a chronic inflammatory bacterial infection leading to destruction of periodontal ligaments and supporting bone of the tooth. Its aetiology has been a field of intensive research in the past decades. As periodontal pockets accommodate a multitude of bacterial phylotypes, it is difficult to differentiate between mere commensals and true pathogens. During the 1970's, 80's and early 90 's, research focused mainly on a number of culturable bacteria like Porphyromonas gingivalis, Prevotella intermedia, Aggregatibacter (Actinobacillus)actinomycetemcomitans, Tannerella forsythia and Treponema

\footnotetext{
* Correspondence: annette.moter@charite.de

${ }^{1}$ Institut für Mikrobiologie und Hygiene, Charité - Universitätsmedizin Berlin, Dorotheenstraße 96, 10117 Berlin, Germany
}

denticola that proved to be associated with the disease [1]. Studies have determined their relative prevalences, interactions and virulence factors [2-7]. By the end of the 1980's, the development of novel, culture-independent techniques allowed the identification of as-yetunculturable and fastidious organisms in patients suffering from periodontitis and added new insight into bacterial communities in periodontal pockets [8-10]. In recent years, research has detected increasing numbers of bacterial species and phylotypes in subgingival plaque and other habitats of the human oral cavity [11-18]. There is little reason to believe that easily culturable bacteria contribute more to the development of periodontitis than fastidious organisms. Doubt has been raised whether the widely accepted periodontal pathogens
C Biomed Central 
P. gingivalis, $P$. intermedia and T. forsythia are appropriate diagnostic markers to differentiate between health and disease $[19,20]$.

Along with these discoveries it became clear that the mere isolation and characterization of bacteria from diseased sites is not a sufficient approach to understand the complex pathogenesis of periodontitis. The organisms do not live in a planktonic form, but rather as a sessile community attached to the tooth surface in a matrix of extracellular polymers [21]. The structure and function of these bacterial biofilms are influenced both by bacterial interactions and host factors. Exploring the biofilm architecture and identifying its bacterial architects are pressing goals in current periodontal research.

Filifactor alocis (ATCC $35896^{\mathrm{T}}$ ) was first isolated in 1985 from the human gingival crevice as Fusobacterium alocis [22] and later reclassified as Filifactor alocis [23]. It is a fastidious, Gram-positive, obligately anaerobic rod that possesses trypsin-like enzymatic activity [24], as do $P$. gingivalis and $T$. denticola $[25,26]$. In recent years, it has been discovered in patients suffering from chronic periodontitis (CP) $[14,18,27,28]$, generalized aggressive periodontitis (GAP) [29] and endodontic infections [30]. Recently, $F$. alocis was detected in elevated numbers in CP patients with periodontal deterioration compared to patients with a stable periodontal condition and was therefore proposed as a potential marker for active disease [19].

The present study chose a DNA-based epidemiological approach utilizing dot blot hybridization to investigate the prevalence of $F$. alocis in subjects with GAP, CP, and in a subject group resistant to periodontitis. Furthermore, fluorescence in situ hybridization (FISH) was employed to analyse the spatial arrangement and the architectural role of $F$. alocis in periodontal pockets. For that purpose, a specially designed carrier system was used to collect in vivo grown biofilms from GAP patients [31].

\section{Methods}

\section{Oligonucleotide probes}

To detect $F$. alocis, a species-specific probe, FIAL (5'TCTTTGTCCACTATCGTTTTGA-3') was designed after comparative sequence analysis of close phylogenetic neighbours to $F$. alocis. To ensure specificity, the probe sequence was compared to the sequences deposited in the Ribosomal Database Project II [32] and to all $16 \mathrm{~S}$ rRNA entries at the EMBL and GenBank databases (as of August 2009) employing the Husar program package (DKFZ, Heidelberg, Germany). The probe was checked for its practical use in hybridization experiments with the program OLIGO (version 4.0). EUB 338, a probe complementary to a highly conserved region of the 16S rRNA gene in bacteria, was used in dot blot hybridization experiments to verify successful PCR amplification and in FISH experiments to detect and visualize large parts of the bacterial biofilm population [33]. For comparative purposes, probes POGI, PRIN, ACAC, TDEN, FUNU and B(T)AFO were employed in dot blot experiments to detect $P$. gingivalis, $P$. intermedia, A. actinomycetemcomitans, T. denticola, Fusobacterium nucleatum and T. forsythia, respectively. These probes have been published previously and deposited in ProbeBase [34].

\section{Clinical samples for dot blot hybridization}

A total of 490 subgingival plaque samples from 121 patients were examined and evaluated. Samples from GAP and CP patients were obtained from those reporting to the departments of periodontology of the Charite - Universitätsmedizin Berlin, the Dresden University of Technology, the University of Oslo and the University of Basel. These patients were diagnosed according to the criteria of the 1999 International Workshop for the Classification of Periodontal Diseases and Conditions [35] (see Table 1). Control samples were taken from elderly patients of a private periodontal practice in Berlin. These subjects, aged 65 years and older, had at least 20 natural teeth and displayed only mild periodontal disease. They had not received periodontal treatment previously, exhibited no sites with attachment loss of more than $2 \mathrm{~mm}$ or probing pocket depth (PPD) of more than $5 \mathrm{~mm}$ and will be referred to as periodontitis resistant (PR) patients in the following. Subjects suffering from chronic systemic disease were excluded from the study as well as pregnant or breast feeding women and patients who had received antiinflammatory or antimicrobial therapy within the past six months. Patient demographics are presented in Table 2. Ethical approval was given by the Ethical Committee at Charité - Universitätsmedizin Berlin. All patients signed informed consent forms. After removal of supragingival plaque the deepest periodontal pockets available were sampled. In GAP patients, additional samples were taken from shallow sites if present. None of the samples were taken from the same site in one patient. Three sterile paper points (ISO 35, Becht, Offenburg, Germany) were inserted into the pockets, removed after 10 seconds and placed immediately in $1 \mathrm{ml}$ of reduced transport fluid (RTF) [36] containing 25\% glucose.

\section{Dot blot hybridization}

DNA extraction from the 490 collected subgingival plaque samples, subsequent PCR amplification, preparation of dot blot membranes and dot blot hybridization experiments to analyse the prevalence of $F$. alocis were performed as 
Table 1 Clinical criteria for patient selection

\begin{tabular}{ll}
\hline Periodontitis Resistant (PR) subjects & Age $\geq 65$ years \\
& $\geq 20$ natural teeth \\
& Probing Depth at any site $\leq 5 \mathrm{~mm}$ \\
& Clinical Attachment Loss at any site $\leq 2 \mathrm{~mm}$ \\
\hline Chronic Periodontitis (CP) & $\geq 4 \mathrm{~mm}$ Probing Depth at $\geq 30 \%$ of residual teeth \\
\hline Generalized Aggressive Periodontitis (GAP) & Disease onset estimated at $<30$ years based on clinical examination, past radiographs, and/or interview \\
& $\geq 6 \mathrm{~mm}$ Probing Pocket Depth at $>3$ permanent teeth other than first molars and incisors \\
\hline
\end{tabular}

Table 2 Patient demographics

\begin{tabular}{|c|c|c|c|c|c|c|}
\hline \multicolumn{7}{|c|}{ Clinical samples processed by dot blot hybridization } \\
\hline \multirow{2}{*}{$\begin{array}{l}\text { Subject } \\
\text { group }\end{array}$} & \multirow{2}{*}{$\begin{array}{c}\text { No. of } \\
\text { patients }\end{array}$} & \multirow{2}{*}{$\begin{array}{l}\text { Age (yr) } \\
\pm S D\end{array}$} & \multicolumn{2}{|c|}{ Gender } & \multicolumn{2}{|c|}{ Plaque samples } \\
\hline & & & f & $\mathrm{m}$ & $\mathbf{n}$ & $\begin{array}{l}\text { mean PPD } \\
(\mathrm{mm}) \pm S D\end{array}$ \\
\hline GAP & 72 & $34.8 \pm 6.4$ & 45 & 27 & 330 & $7.8 \pm 2.5$ \\
\hline $\mathrm{CP}$ & 30 & $51.0 \pm 10.2$ & 15 & 15 & 78 & $7.1 \pm 1.4$ \\
\hline PR & 19 & $66.7 \pm 1.5$ & 12 & 7 & 82 & $3.6 \pm 0.8$ \\
\hline \multicolumn{7}{|c|}{ Clinical samples for FISH } \\
\hline \multirow[t]{2}{*}{$\begin{array}{l}\text { Subject } \\
\text { group }\end{array}$} & $\begin{array}{l}\text { No. of } \\
\text { patients }\end{array}$ & $\begin{array}{l}\text { Age }(y r) \\
\pm S D\end{array}$ & \multicolumn{2}{|c|}{ Gender } & \multicolumn{2}{|c|}{ Carrier samples } \\
\hline & & & $f$ & $\mathrm{~m}$ & $\mathrm{n}$ & $\begin{array}{l}\text { mean PPD } \\
(\mathrm{mm}) \pm \mathrm{SD}\end{array}$ \\
\hline GAP & 11 & $34.3 \pm 7.9$ & 5 & 6 & 28 & $8.1 \pm 1.7$ \\
\hline
\end{tabular}

published previously [37]. The broad range bacterial primers TPU1 5'-AGAGTTTGATCMTGGCTCAG-3' (corresponding to complementary positions $8-27$ in the Escherichia coli 16S rRNA gene) and RTU3 5'-GWATTACCGCGGCKGCTG-3' (corresponding to positions 519536 in E. coli $16 \mathrm{~S}$ rRNA) were used to amplify part of the $16 \mathrm{~S}$ rRNA gene out of the bulk DNA. Agarose gel electrophoresis confirmed successful amplification. Hybridizations with both EUB 338 and FIAL were carried out at $54^{\circ} \mathrm{C}$, while stringency washes were performed at $58^{\circ} \mathrm{C}$ for EUB 338 and at $60^{\circ} \mathrm{C}$ for FIAL with a washing buffer containing $2 \times \mathrm{SSC}(1 \times \mathrm{SSC}$ is $0.15 \mathrm{M} \mathrm{NaCl}$ plus $0.015 \mathrm{M}$ sodium citrate) - 0.1\% SDS for EUB 338 and 5x SSC - 0.2\% SDS for FIAL. In all experiments, PCR-amplified products obtained from fixed cells of $F$. alocis, its closest cultured phylogenetic relative Filifactor villosus (ATCC $33388^{\mathrm{T}}$ ), and a panel of 43 periodontal pathogens (see Figure 1 legend) and related bacteria were included as positive and negative controls, respectively. After hybridization, X-ray films were exposed for 2 to 30 hours. After stripping, all membranes were reused for further experiments.

\section{Statistical analysis}

Statistical evaluation of the dot blot hybridization results was performed using the exact chi-square test. The prevalence of $F$. alocis in different patient groups was compared. Moreover, the presence of $F$. alocis in relation to the PPD was analysed. P values below 0.05 were considered statistically significant.

\section{Clinical samples for FISH}

A carrier system designed to collect biofilms grown in vivo in periodontal pockets was used for sampling [31]. Ethics approval for subgingival sample collection was given by the Ethical Committee at Charité - Universitätsmedizin Berlin. Expanded polytetrafluoroethylene (ePTFE) membranes were placed in periodontal pockets of GAP patients for 7 to 14 days and colonized by the subgingival bacterial flora. Strips of ePTFE measuring $3 \mathrm{~mm}$ in width were wrapped around and attached to rigid plastic tips (Plast-O-Probe; Maillefer, Ballaigues, Switzerland), which permitted the insertion of these strips down to the bottom of the pocket, therefore allowing the complete extension of the membrane over the entire probing depth. One side of the double bent strip faced the soft tissue and the other side, slightly longer, faced the root surface. This longer cervical end was fixed to the tooth with cyanoacrylic glue (Tesa, Beiersdorf, Hamburg, Germany) to stabilize the position of the carrier. After removal, carriers were fixed for at least $3 \mathrm{~h}$ with $3.7 \%(\mathrm{v} / \mathrm{v})$ formaldehyde in phosphatebuffered saline ( $\mathrm{pH}$ 7.4) and embedded in cold polymerizing resin (Technovit 8100, Kulzer, Wehrheim, Germany) as reported previously [38]. Sectioning into slices of 2-3 $\mu \mathrm{m}$ was performed as previously published [39]. A total of 28 carriers from 11 GAP patients seeking treatment at the Charité - Universitätsmedizin Berlin were examined. These patients met the same inclusion criteria as the GAP patients selected for dot blot hybridization and likewise signed informed consent forms. See Table 2 for patient demographics. Additionally, a gingival biopsy of a GAP patient obtained during periodontal surgery was processed in the same manner and included in the FISH experiments.

\section{FISH}

FISH experiments were performed as described previously [40] apart from using Vectashield containing DAPI (4,6-Diamidino-2-Phenylindoldihydrochlorid) (Vector Laboratories, Orton Southgate, UK) as mounting medium. The probes were synthesized commercially 

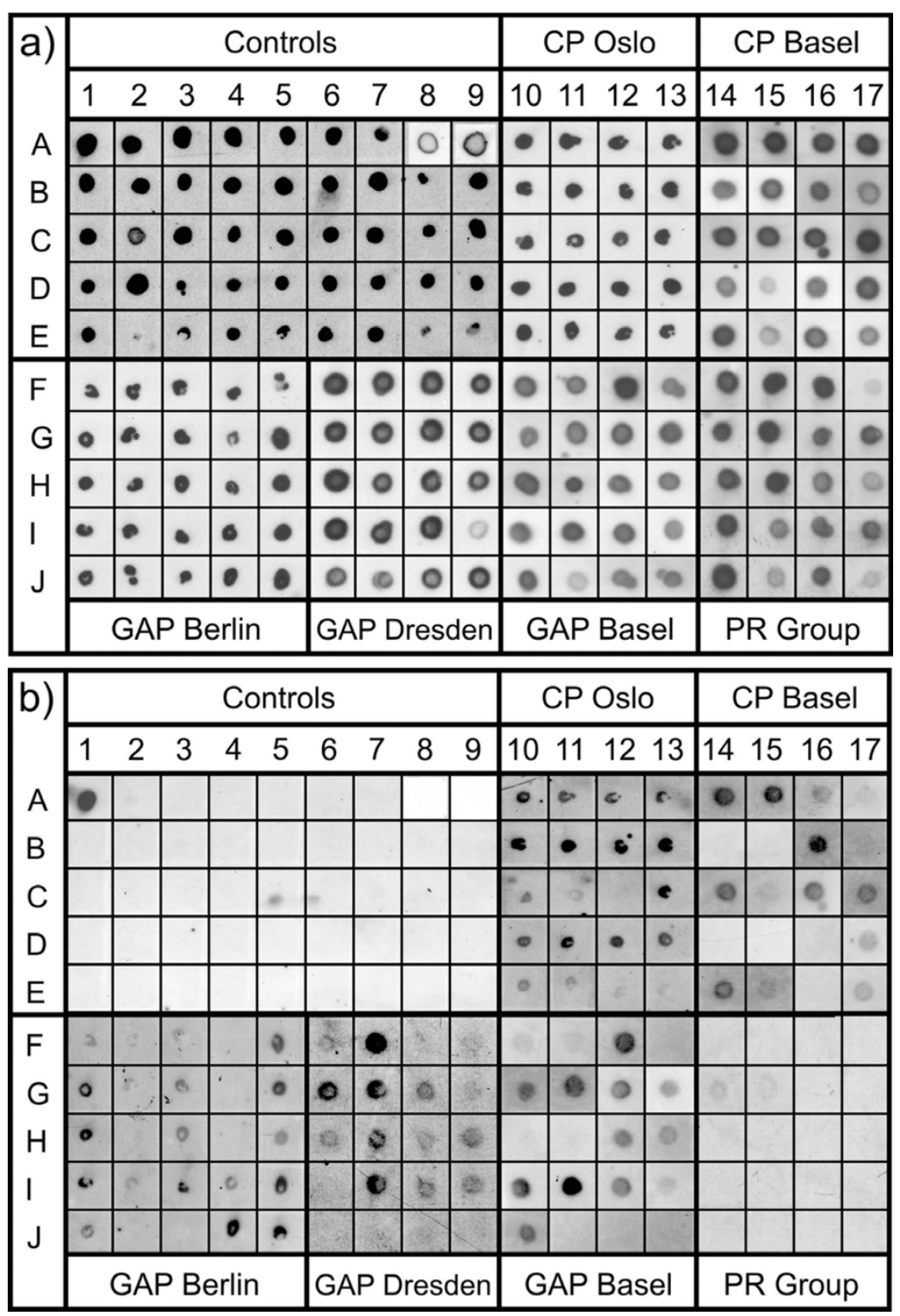

Figure 1 Dot blot hybridizations of identical membranes with EUB 338 (a) and the species-specific probe FIAL (b). PCR-amplified products from F. alocis (field A1) and its closest cultured relative F. villosus (A2) served as positive and negative controls, respectively. Additionally, products from the following bacteria were applied as negative controls: Centipeda periodontii (DSM 2778) (A3), Selenomonas noxia (DSM 19578) (A4), Selenomonas ruminantium (DSM 2150) (A5), Selenomonas lacticifex (DSM 20757) (A6), Selenomonas sputigena (DSM 20758) (A7), Eggerthella lenta (ATCC 25559) (A8), Peptostreptococcus anaerobius (ATCC 27337) (A9), and Actinomyces viscosus (ATCC 15987) (B1), Streptococcus intermedius (ATCC 27335) (B2), Streptococcus mutans (ATCC 35668) (B3), Neisseria lactamica (ATCC 23970) (B4), Flavobacterium odoratum (ATCC 4651) (B5), Fusobacterium necrophorum (NCTC 25286) (B6), Fusobacterium periodonticum (CCUG 14345) (B7), Fusobacterium simiae (CCUG 16798) (B8), F. nucleatum (ATCC 25586) (B9), Klebsiella pneumoniae (ATCC 23357) (C1), Veillonella dispar (ATCC 17748) (C2), Veillonella parvula (ATCC 10790) (C3), Kingella kingae (ATCC 23330) (C4), Eikenella corrodens (CCUG 2138) (C5), Bacteroides fragilis (ATCC 25285) (C6), Bacteroides gracilis (ATCC 33236) (C7), Campylobacter concisus (ATCC 33236) (C8), Campylobacter rectus (ATCC 33238) (C9), Capnocytophaga gingivalis (ATCC 33624) (D1), Capnocytophaga sputigena (ATCC 33612) (D2), Capnocytophaga ochracea (ATCC 27872) (D3), Prevotella buccalis (ATCC 33690) (D4), Prevotella oralis (MCCM 00684) (D5), Prevotella nigrescens (NCTC 9336) (D6), Porphyromonas asaccharolytica (ATCC 25260) (D7), P. intermedia (ATCC 25611) (D8), P. gingivalis (ATCC 33277) (D9), Haemophilus paraphrophilus (ATCC 29241) (E1), Haemophilus aphrophilus (NCTC 55906) (E2), Haemophilus influenzae (clinical isolate) (E3), Haemophilus influenzae (ATCC 33391) (E4), Pasteurella haemolytica (ATCC 33396) (E5), Leptotrichia buccalis (MCCM 00448) (E6), A. actinomycetemcomitans (MCCM 02638) (E7), A. actinomycetemcomitans (ATCC 33384) (E8) and A. actinomycetemcomitans (ATCC 43718) (E9). In columns 10-17 and in lanes F to J of columns 1-9 PCR products from patient samples of the different diseased groups and the periodontitis resistant (PR) group were applied. (a): Signals in all fields prove successful PCR-amplification. (b): Absence of signals in all bacterial controls along with strong signal in field A1 proves specificity of the experiments. Prevalences of $F$. alocis in all diseased collectives exceed the prevalence in the PR group. 
(biomers.net, Ulm, Germany). EUB 338 was 5' endlabelled with fluorochrome Cy5 (indodicarbocyanine) while FIAL was 5' end-labelled with fluorochrome $\mathrm{Cy} 3$ (indocarbocyanine). Differential labelling allowed simultaneous hybridization with both probes.

\section{Optimization of probe FIAL for FISH}

The stringency of FIAL was adjusted by incubating fixed cells of $F$. alocis and its closest cultured relative, F. villosus with different hybridization mixes. The formamide concentrations covered a range from $0 \%(\mathrm{v} / \mathrm{v})$ to $75 \%$ $(\mathrm{v} / \mathrm{v})$, rising in steps of $5 \%(\mathrm{v} / \mathrm{v})$. At each level of formamide, a series of images of each bacterial species was taken with a fixed exposure time. The software daime [41] was used to measure the light intensities emitted by both species for each concentration of formamide. While the signal intensity of $F$. villosus did not reach 50 Relative fluorescence Units (RU) at any level of formamide due to unspecific binding of the probe, the intensity of $F$. alocis remained constantly above $150 \mathrm{RU}$ using formamide concentrations of up to $20 \%(\mathrm{v} / \mathrm{v})$ (see Additional file 1). In addition, fixed cells of 16 different bacterial species, most of them periodontal pathogens, were incubated with FIAL at $20 \%(\mathrm{v} / \mathrm{v})$ formamide as negative controls, namely $F$. nucleatum (ATCC 25586), Eikenella corrodens (CCUG 2138), Kingella kingae (ATCC 23330), Veillonella parvula (ATCC 10790), Veillonella dispar (ATCC 17748), P. gingivalis (ATCC 33277), A. actinomycetemcomitans (ATCC 33384), Pasteurella haemolytica (ATCC 33396), T. forsythia (ATCC 43037), Haemophilus aphrophilus (NCTC 55906) P. intermedia (ATCC 25611), Campylobacter rectus (ATCC 33238), Capnocytophaga sputigena (ATCC 33612), Capnocytophaga gingivalis (ATCC 33624), Eggerthella lenta (ATCC 25559), and Peptostreptococcus anaerobius (ATCC 27337). As none of the controls were detected by FIAL, all further experiments were performed with $20 \%(\mathrm{v} / \mathrm{v})$ of formamide, including $F$. alocis as positive and $F$. villosus as negative control.

\section{Epifluorescence microscopy}

After hybridization, carrier and biopsy sections were analysed using an epifluorescence microscope (AxioPlan II, Zeiss, Jena, Germany) equipped with a $100 \mathrm{~W}$ high pressure mercury lamp (HBO $103 \mathrm{~W} / 2$, Osram, Munich, Germany) and $10 \times, 40 \times$ and $100 \times$ objectives. DAPI, Cy3 and Cy5 signals were analysed by narrow band filter sets HQ F31-000, HQ F41-007 and HQ F41-008, respectively (AHF Analysentechnik, Tübingen, Germany). Image acquisition was performed with an AxioCam MRm (Zeiss) making use of the AxioVision 4.4 software.

\section{Results}

\section{Dot blot hybridization}

When carried out with the probe EUB 338 (specific for most bacteria), dot blot hybridization experiments indicated the presence of bacteria in all 490 patient samples as well as in the positive (F. alocis) and negative controls (see Figure 1 legend) and thus confirmed successful PCR amplification (Figure 1a). The Filifactor alocis-specific probe FIAL clearly detected $F$. alocis, while neither the closest phylogenetic neighbour $F$. villosus nor any of the organisms in the panel of oral bacteria (see Figure 1 legend) yielded a signal, thus indicating specific hybridization conditions (Figure 1b).

Taking all the collected samples into consideration, $F$. alocis could be identified in $77.8 \%$ of the 330 samples from 72 GAP patients, $76.7 \%$ of the 78 samples from 30 $\mathrm{CP}$ patients and $15.8 \%$ of the 82 samples from $19 \mathrm{PR}$ patients (Table 2; Figure 2a). The prevalence of the organism was highest in the Oslo CP collective (87.5\%), followed by the Basel GAP collective $(80.0 \%)$, and the Dresden GAP collective (77.8\%) (data not shown). As the number of samples per patient varied between the different collectives, statistical evaluation focused on the deepest pocket of each patient. Prevalence rates were 68.1\% for the GAP group, $66.7 \%$ for the CP group and $5.3 \%$ for the PR group. While detection frequencies did not differ significantly between GAP and CP patients, both diseased groups harboured $F$. alocis significantly more often than the PR group ( $<$ 0.001) (Figure $2 b$ ).

The signal intensity of the FIAL-positive patient samples varied between the three groups, suggesting a higher number of Filifactor in GAP and CP pockets than in PR pockets tested positive for the organism. Nonetheless, as hybridizations were carried out on PCRamplified bacterial DNA, no further analysis of signal intensities was performed.

Detection frequencies of $P$. gingivalis, $P$. intermedia, A. actinomycetemcomitans, $T$. denticola, $T$. forsythia, and $F$. nucleatum in the three patient groups are displayed in Figure 2b.

To investigate the prevalence of $F$. alocis in relation to the PPD, the donor sites were divided into four groups (I: $1-3 \mathrm{~mm}$, II: 4-6 mm, III: 7-9 mm, IV: > $9 \mathrm{~mm}$ ). As there is a certain degree of interdependency between pockets belonging to the same patient, statistical analysis was limited to one pocket per patient and probing depth group. Although a slightly higher percentage of group III pockets than group II pockets was positive for Filifactor in both the GAP and the CP patients, these differences were not statistically significant. Similarly, analysis revealed no statistically significant differences in the prevalence of the organism in GAP patients compared to 


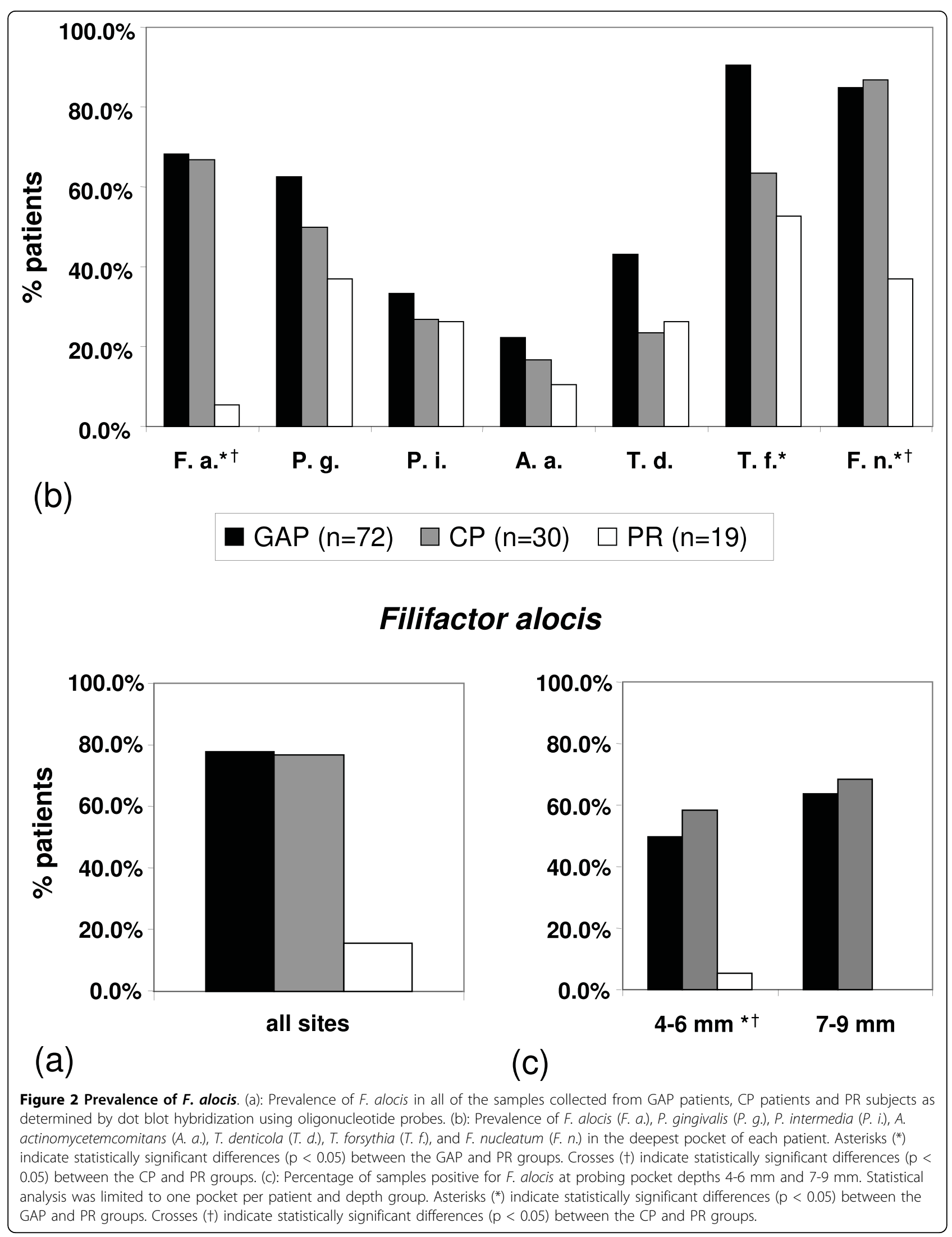


CP patients in both pockets of 4-6 $\mathrm{mm}$ and pockets of 7-9 $\mathrm{mm}$. In contrast, the prevalence of $F$. alocis in pockets of 4-6 mm differed significantly between both PR and GAP patients $(\mathrm{p}<0.001)$ and $\mathrm{PR}$ and $\mathrm{CP}$ patients $(\mathrm{p}<0.001)$ (Figure 2c). Insufficient numbers or complete absence of pockets of 1-3 $\mathrm{mm}$ in GAP and CP patients, pockets of 7-9 $\mathrm{mm}$ in $\mathrm{PR}$ patients and pockets deeper than $9 \mathrm{~mm}$ in $\mathrm{CP}$ and PR patients did not permit further statistical analysis.

\section{FISH}

F. alocis was reliably detected by both the species-specific probe FIAL and the eubacterial probe EUB 338. The negative control F. villosus was not targeted by FIAL but only by EUB 338, thus confirming specific hybridization conditions (Figure 3 ). In all of the periodontal ePTFE carriers from GAP patients as well as in the gingival biopsy gained during periodontal surgery, the bacterial biofilms could be visualized by FISH with EUB 338 and displayed characteristic features like densely- packed mushroom-like protuberances and signal-free channels [42]. F. alocis could be detected in 9 out of 11 carrier patients (in 17 out of 28 carriers) as well as in the examined gingival biopsy.

In the carrier-grown biofilms, the organism could be visualized in those areas that had grown in the depth of the pocket, but rarely in areas corresponding to the cervical part of the pocket and rarely on the very tip of the carrier. In most cases, Filifactor colonized the side of the carrier facing the soft tissue (Figure 4c) and could only be found in few numbers or not at all on the carrier side facing the root (Figure $4 \mathrm{~b}$ ). Many parts of the biofilm showed $F$. alocis as a short rod of 1-2 $\mu$ m length, whereas at some sites the organism appeared longer, extending to 7-8 $\mu \mathrm{m}$ (Figure $5 \mathrm{a}$ ). While in some areas Filifactor cells seemed to be scattered within the biofilm without any recognizable pattern, numerous sites clearly showed a higher degree of organisation. Repeatedly, F. alocis could be found in densely packed groups (Figure $4 c$ ), arranged in concentrical structures (Figure $5 \mathrm{~d}$ )

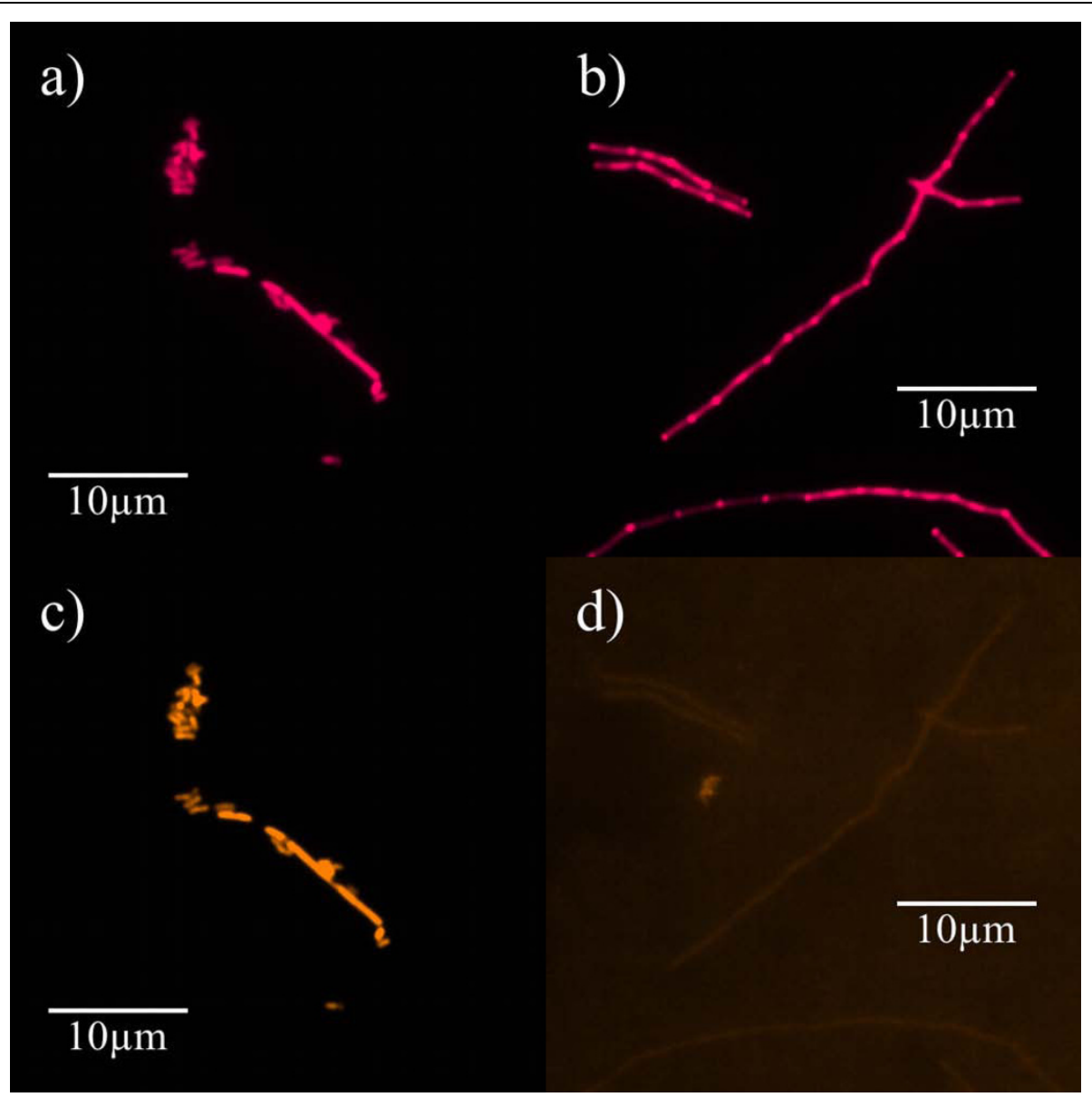

Figure 3 Specificity of FISH experiments. Hybridization of fixed cells of F. alocis (a and c) and F. villosus ( $b$ and d) was performed with probes EUB 338-Cy5 (magenta, $a$ and b) and FIAL-Cy3 (bright orange, $c$ and d). (a and c): Identical microscopic fields show detection of F. alocis by both EUB 338 (a) and FIAL (c) whereas detection of F. villosus by EUB 338 only (b) and not FIAL (d) proves specificity of the FISH experiment. 


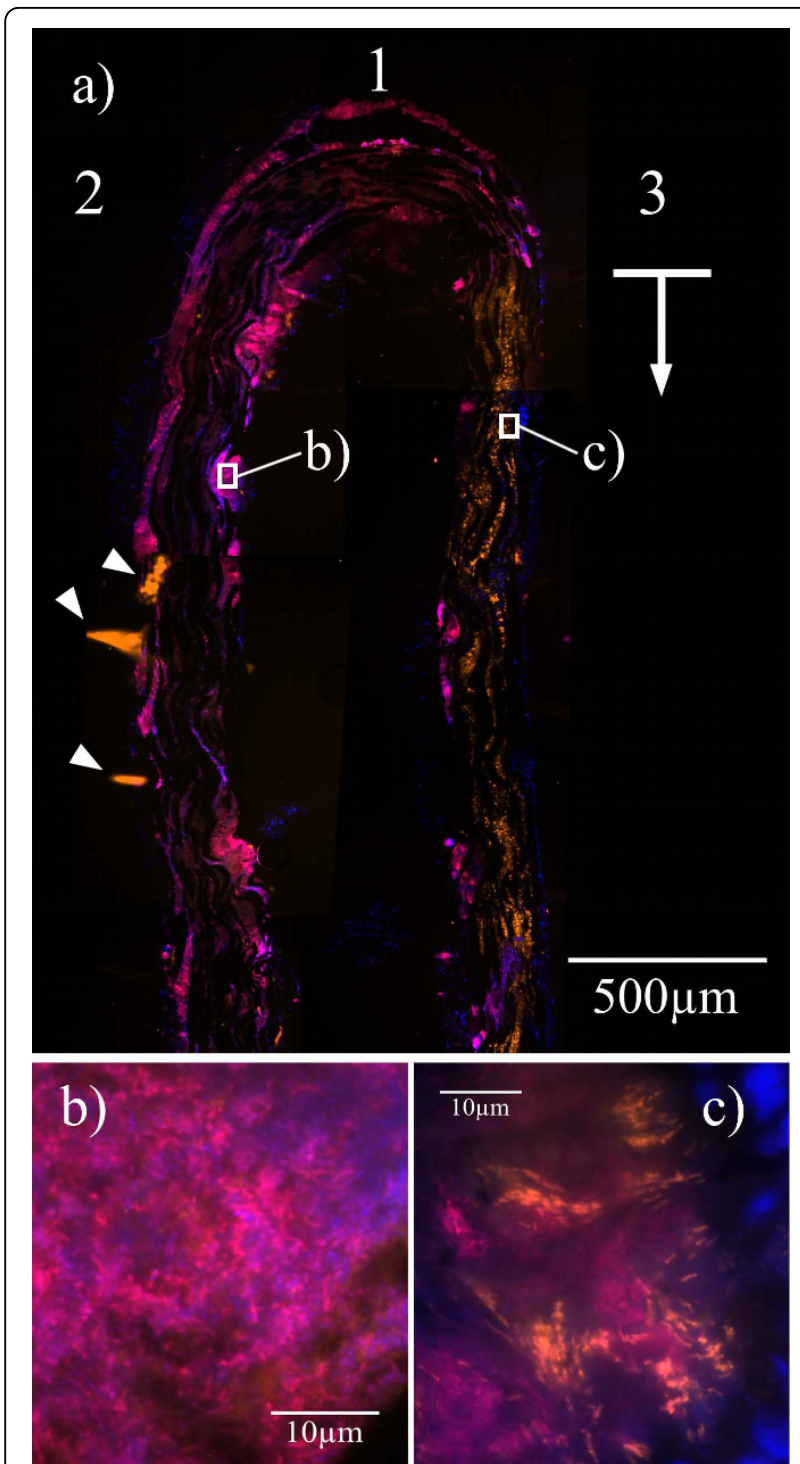

Figure 4 Carrier grown biofilm visualized by FISH. Hybridization was performed with the probes EUB 338-Cy5 (magenta) and FIALCy3 (bright orange) along with DAPI staining (blue) on a carrier after 7 days of attachment to the mesial aspect of tooth 16 in a GAP patient. (a): Collage of several microscopic fields in low magnification. The overlay of Cy3, Cy5 and DAPI filter sets shows the bacterial biofilm that grew in the depth of the pocket. EUB 338 visualizes large parts of the bacterial community, while FIAL detects only F. alocis. DAPI stains both host cell nuclei and bacteria. The carrier tip (1) and the carrier side facing the tooth (2) show little or no presence of $F$. alocis. The bright orange signal on the carrier side facing the pocket epithelium (3) reveals a strong presence of Filifactor in the part of the biofilm indicated by the arrow. Arrowheads on the tooth side (2) point to artifacts caused by upfolding of the embedded carriers. ( $b$ and $c$ ): Higher magnifications of the inserts. (b) shows the biofilm on the tooth side of the carrier without $F$. alocis among the bacteria. (c) shows F. alocis in densely packed groups among the organisms on the epithelium side and host cell nuclei (blue). or grouped in "test-tube brush" formations [43] around signal free channels (Figure 5c). Figure 5b shows the radial orientation of $F$. alocis towards the surface of a mushroom-like protuberance of the biofilm.

Similar formations that indicate ultrastructural organisation of the biofilm could be observed in the gingival biopsy. In several areas, $F$. alocis formed branch-like structures within the affected tissue (Figure 6a) or palisades around large rodshaped bacteria (Figure $6 \mathrm{~b}$ ). Again, Filifactor was observed among the organisms in concentric bacterial aggregations (Figure 6c).

\section{Discussion}

To our knowledge, the present study is the first to analyse the prevalence of $F$. alocis in samples from both GAP and CP patients, and subjects with apparent periodontitis resistance. The detection of the organism in $77.8 \%$ of the GAP patients and in $76.7 \%$ of those suffering from $\mathrm{CP}$ is convincing evidence that suggests an involvement of $F$. alocis in periodontal disease. Equally striking is the low prevalence of Filifactor in the PR group. All of these patients had reached the age of 65 years and were in good periodontal condition without the help of extensive therapeutic efforts. Even if a multitude of factors including oral hygiene and immune response contributed to their periodontal status, one would assume that frequent detection of an organism in the GAP and CP groups along with scarce detection in PR patients, as is the case for F. alocis, indicates pathogenic rather than commensal behaviour.

One can argue that deep periodontal pockets harbour increased numbers of bacteria and that any organism inevitably should be isolated more constantly from CP patients (mean pocket depth: $7.13 \mathrm{~mm}, 1.4 \mathrm{~mm} \mathrm{SD}$ ) and especially GAP patients $(7.81 \mathrm{~mm}, 2.48 \mathrm{~mm} \mathrm{SD})$ than from PR patients $(3.63 \mathrm{~mm}, 0.79 \mathrm{~mm} \mathrm{SD})$. However, dividing the entirety of the sampled sites into four groups according to the measured PPD (I: $1-3 \mathrm{~mm}$, II: 4-6 mm, III: 7-9 mm, IV: > $9 \mathrm{~mm}$ ), statistical analysis of pockets between 4 and $6 \mathrm{~mm}$ still reveals a significantly higher prevalence of $F$. alocis in both the GAP and the $\mathrm{CP}$ group compared to the PR group. In addition, the organism was not detected significantly more frequently in deeper pockets $(7-9 \mathrm{~mm})$ than in rather shallow pockets $(4-6 \mathrm{~mm})$ in both GAP and $\mathrm{CP}$ patients. Although a connection between PPD and bacterial load cannot be denied, these findings indicate that the influence of pocket depth does not invalidate the aforementioned results.

If one compares the prevalence rate of $F$. alocis to those of the widely accepted periodontal pathogens $P$. gingivalis, $P$. intermedia, A. actinomycetemcomitans, 


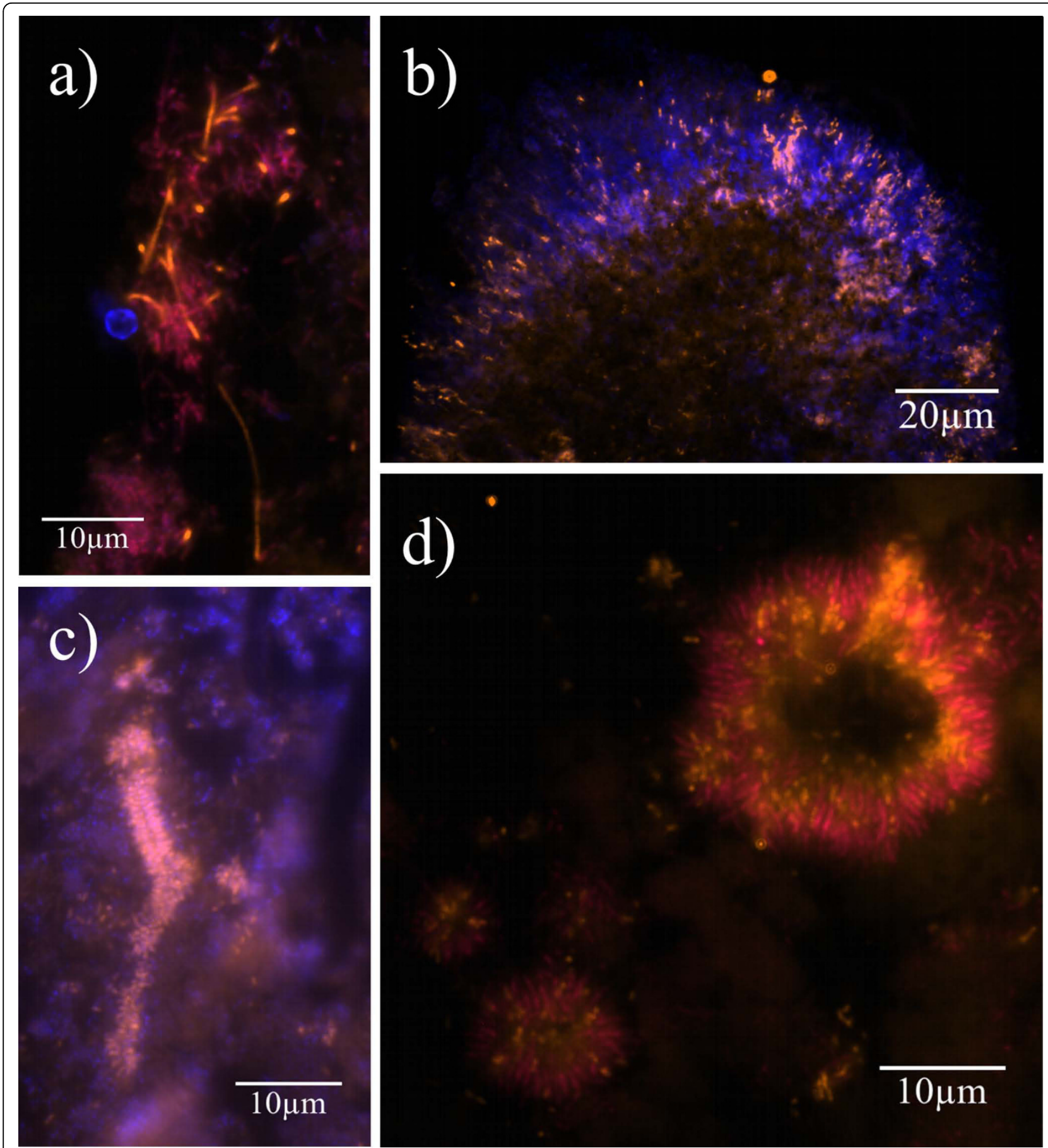

Figure 5 Formations of $\boldsymbol{F}$. alocis in carrier-borne biofilms. FISH on different carriers with GAP biofilms using the probes EUB $338-C y 5$ (magenta) and FIAL-Cy3 (bright orange) along with DAPI staining (blue). EUB 338 detects the whole bacterial population while FIAL visualizes F. alocis specifically. DAPI stains both bacteria and host cell nuclei. High magnifications show F. alocis in different areas of the biofilms. (a): Overlay of Cy3, Cy5 and DAPI filter sets. In some regions of the biofilm Filifactor rods can reach a considerable length. (b and c): Overlay of Cy3 and DAPI filter sets. (b) shows the radial orientation of $F$. alocis and other organisms on the surface of a mushroom-like protuberance of the biofilm. (c) shows F. alocis forming test-tube-brush-like structures around a signal-free channel. (d): Overlay of Cy3 and Cy5 filter sets. F. alocis and fusiform bacteria form concentrical structures. 


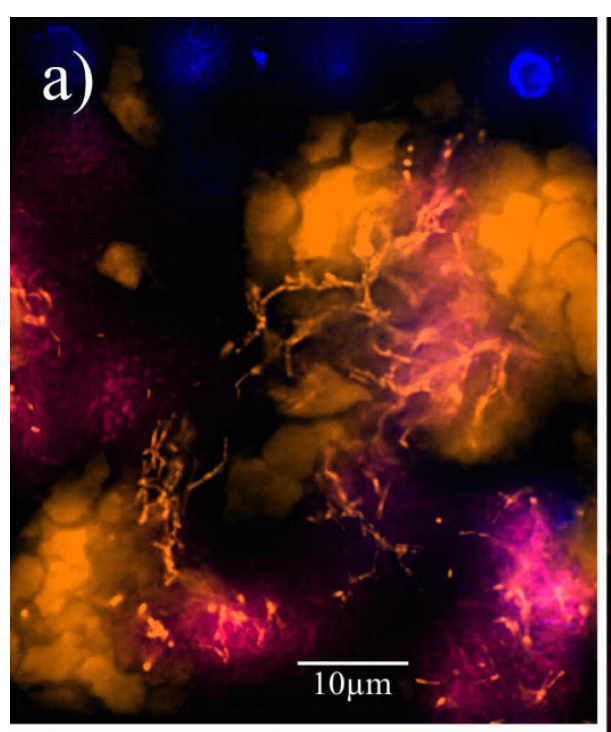

\section{b)}
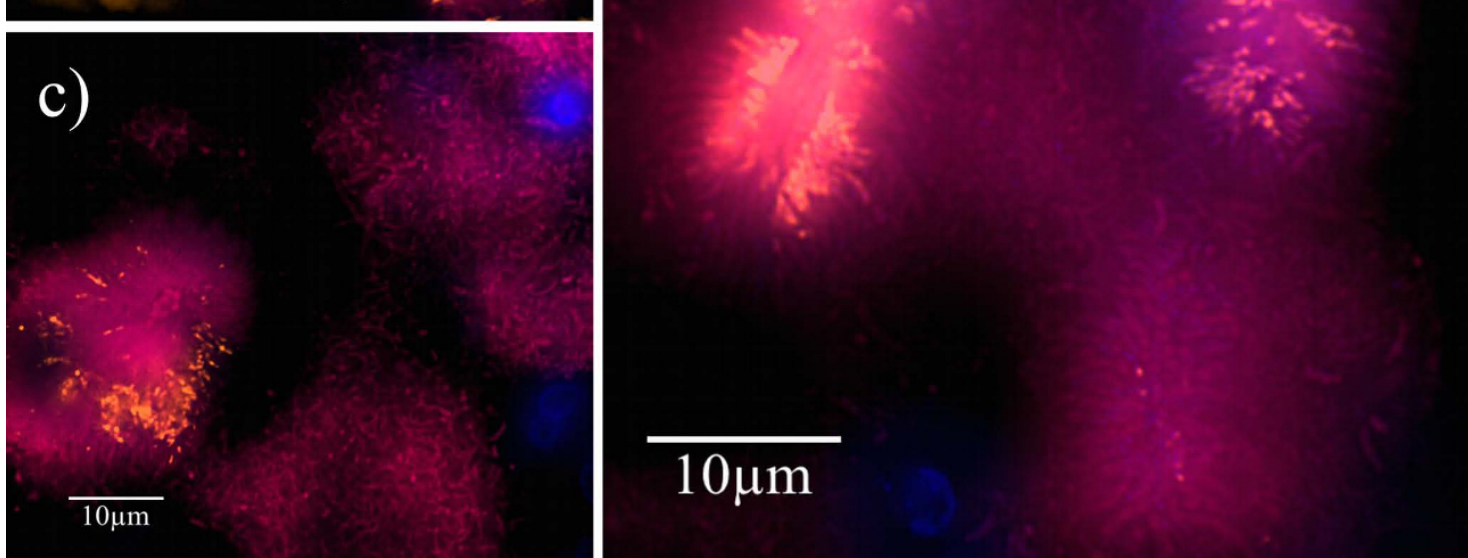

Figure 6 Formations of $\boldsymbol{F}$. alocis in periodontal tissue. FISH on a biopsy gained during periodontal surgery using the probes EUB 338-Cy5 (magenta) and FIAL-Cy3 (bright orange) along with DAPI staining (blue). EUB 338 visualizes the entire bacterial community, while FIAL detects only F. alocis. DAPI stains both host cell nuclei and bacteria. High magnifications depict F. alocis in different parts of the biopsy. (a): $F$. alocis forms tree-like structures among coccoid and fusiform bacteria and autofluorescent erythrocytes. (b) shows $F$. alocis forming palisades with fusiform bacteria around large rodshaped eubacterial organisms. (c) shows F. alocis being part of concentrical bacterial aggregations resembling those detected in GAP carriers.

T. denticola, F. nucleatum, and T. forsythia (see Figure $2 \mathrm{~b})$, investigated in these very samples using identical methods, Filifactor is the third most prevalent for GAP and second most prevalent for $\mathrm{CP}$ patients and is thus at eye level with organisms that are considered key players in periodontal disease. At the same time, F. alocis shows the lowest prevalence in the PR group of all analysed organisms. Together with F. nucleatum, F. alocis is the only organism to show a significantly higher detection frequency in both GAP and CP patients compared to the PR group.

Using PCR-based identification methods may introduce bias, since structurally different organisms could exhibit different copy numbers of ribosomal genes and will generally respond differently to DNA isolation and the chosen set of broad range bacterial primers [44].
However, the relevance of $F$. alocis is supported by several other epidemiological studies conducted in the past years using DNA-based techniques. $F$. alocis was detected in GAP patients as well as in CP patients with prevalence rates varying between $45 \%$ [29] and 90\% [28], depending on the methods employed. Some authors propose $F$. alocis as a marker organism for periodontal disease [28] and even for the shift from periodontal health to disease [19].

Our data strongly support the findings of these studies and motivated the attempt to visualize $F$. alocis within the periodontal biofilm of GAP patients using FISH. The organism could be detected in high numbers in the majority of the examined carriers. The percentage of positive patients approximately matches the dot blot results. Strikingly, several areas of the biofilm show 
F. alocis in densely packed groups (Figure 4c) or as a part of concentric bacterial agglomerations (Figure 5d) formations that suggest a certain degree of organisation to the observer. Moreover, the organism could be visualized in structures that are considered characteristic architectural features of periodontal biofilms. F. alocis is among the bacteria in mushroom-like protuberances on the surface of the biofilm (Figure $5 \mathrm{~b}$ ) and it contributes, grouped around what might be diffusion or convection channels, to the formation of structures reminding of test-tube brushes (Figure 5c). The close colocalization of F. alocis with other periodontal pathogens suggests that Filifactor might be involved in coaggregation events that take place during the establishment and maturation of the biofilms and that are thought to play a crucial role in biofilm formation [45]. Moreover, the tight colocalization might indicate necessary symbiotic relationships that could help to explain the fastidiousness of Filifactor.

Just like group I treponemes [31], F. alocis predominantly colonizes the apical and middle third of the carriers and could only casually be detected in the cervical third. Most interestingly, the organism preferably settles on the side of the carrier facing the soft tissues and is thus in immediate contact to the host's immune defence. All these observations point to a causal involvement of $F$. alocis in the formation and maintenance of the analysed biofilms.

However, one might question whether these carrierborne biofilms accurately model the unperturbed biofilms in periodontitis patients. Wecke et al. [31] compared the bacterial load after 3 and 6 days and showed that the biofilm mass covering the carriers increases with time. The presence of $F$. alocis on only one side of the membranes is further evidence that these samples are not simply fragments of biofilm torn out of the pocket during the removal of the carriers, but in fact newly grown biofilms that form while the carriers are in situ. Although FISH reveals structural elements specific to periodontal biofilms, one cannot deny that the introduction of the carrier into the periodontal pocket creates an artificial environment. The barrier between root surface and pocket epithelium might hamper access of the immune system to the bacteria on the tooth side, while only the biofilm growing on the soft tissue side actually faces the host. Moreover, these biofilms do not form on natural substrate but instead on ePTFE membranes. However, it seems likely that the substrate is of minor importance to the biofilm development. Wecke et al. [31] did not observe differences between biofilms grown on different carrier materials, and it is likely that the acquired pellicle, which covers both the root and the membrane, renders colonization conditions on a broad range of materials alike. This claim is supported by microscopic examination of the biopsy submitted to
FISH. F. alocis could be visualized in high numbers and detected in arrangements similar to those seen in carrier-borne biofilms. Thus, a contribution of Filifactor to the structural organisation of 'naturally' grown biofilms seems highly probable.

The applied carrier system proves to be a valuable tool for the exploration of periodontal biofilms as it allows to investigate topographic relations within the pocket without invasive treatment. Subsequent FISH permits to analyse the distribution and colocalization of potential pathogens within the biofilm and can thus contribute to a better understanding of the complex host-microbe interactions that lead to periodontal destruction.

\section{Conclusions}

The prevalence of Filifactor alocis in both GAP and CP patients was found to be elevated as compared to PR control. F. alocis thus seems to be a powerful diagnostic marker organism for periodontal disease. FISH revealed the involvement of $F$. alocis in numerous structural arrangements that point to its potential role as one of the architects of structural organisation within periodontal biofilms. Filifactor alocis should be considered an important periodontal pathogen and warrants further research.

\footnotetext{
Additional file 1: Optimization of probe FIAL for FISH using the program daime. FISH was performed incubating fixed cells of $F$. alocis and $F$. villosus with different hybridization mixes. Signal intensities (Relative fluorescent Units, RU) emitted by F. alocis and F. villosus at different formamide concentrations were calculated from images taken with a fixed exposure time. Due to unspecific binding of FIAL, the light emission of $F$. villosus cells remained below $50 \mathrm{RU}$ at every level of formamide. The signal emitted by $F$. alocis cells was considered sufficient using formamide concentrations of up to $20 \%(\mathrm{v} / \mathrm{v})$. Click here for file

[http://www.biomedcentral.com/content/supplementary/1471-2180-1066-S1.PPT]
}

\section{Acknowledgements}

We thank Eva Kulik, University of Basel, and Eivind Strøm, University of Oslo, for providing clinical samples, Cindy Hefenbrock and Marie Knüver for excellent technical assistance, Derek Ramsey for proof reading, and Dr. WolfUlrich Klotz for his support.

This work was supported by the Sonnenfeld-Stiftung, Berlin, Germany, and by a Rahel-Hirsch grant from Charité - Universitätsmedizin to AM.

\section{Author details}

${ }^{1}$ Institut für Mikrobiologie und Hygiene, Charité - Universitätsmedizin Berlin, Dorotheenstraße 96, 10117 Berlin, Germany. ${ }^{2}$ Abteilung Parodontologie, Centrum für Zahn-, Mund- und Kieferheilkunde, Charité - Universitätsmedizin Berlin, Aßmannhauser Straße 4-6, 14197 Berlin, Germany. ${ }^{3}$ Section of Pediatric Dentistry, College of Dentistry, Ohio State University, 305 W. 12th Avenue, Columbus, Ohio 43210, USA.

\section{Authors' contributions}

SS assisted in designing the study, designed and optimized the oligonucleotide probe FIAL, participated in patient sample preparation, carried out dot blot and fluorescence in situ hybridizations, evaluated the data and drafted the manuscript. BR collected patient samples for dot blot 
hybridization, performed statistical analysis and helped to draft the manuscript. ALG provided the initial idea and participated in designing the study. AP participated in patient sample preparation, dot blot hybridizations and FISH probe optimization. $\mathrm{JH}$ provided the gingival biopsy, participated in patient sample preparation and FISH experiments. MB assisted in probe design and dot blot hybridizations. AF developed the periodontal carriers and collected subgingival biofilms for FISH experiments. UBG was involved in designing the study and supervised the work. AM designed and supervised the study and the experiments, analysed the data and participated in writing. All authors read and approved the final manuscript.

Received: 21 August 2009 Accepted: 1 March 2010

Published: 1 March 2010

\section{References}

1. Haffajee AD, Socransky SS: Microbial etiological agents of destructive periodontal diseases. Periodontol 2000 1994, 5:78-111.

2. Kolenbrander PE, London J: Adhere today, here tomorrow: oral bacterial adherence. J Bacteriol 1993, 175:3247-3252.

3. Dahlen GG: Black-pigmented gram-negative anaerobes in periodontitis. FEMS Immunol Med Microbiol 1993, 6:181-192.

4. Fives-Taylor PM, Meyer DH, Mintz KP, Brissette C: Virulence factors of Actinobacillus actinomycetemcomitans. Periodontol 2000 1999, 20:136-167.

5. Cutler CW, Kalmar JR, Genco CA: Pathogenic strategies of the oral anaerobe, Porphyromonas gingivalis. Trends Microbiol 1995, 3:45-51.

6. Sela MN: Role of Treponema denticola in periodontal diseases. Crit Rev Oral Biol Med 2001, 12:399-413.

7. Slots J, Listgarten MA: Bacteroides gingivalis, Bacteroides intermedius and Actinobacillus actinomycetemcomitans in human periodontal diseases. J Clin Periodontol 1988, 15:85-93.

8. Murray PA, French CK: DNA probe detection of periodontal pathogens. New biotechnology in oral research Basel: KargerWM M 1989, 33-53.

9. Chuba PJ, Pelz K, Krekeler G, de Isele TS, Gobel U: Synthetic oligodeoxynucleotide probes for the rapid detection of bacteria associated with human periodontitis. J Gen Microbiol 1988, 134:1931-1938.

10. Conrads G: DNA probes and primers in dental practice. Clin Infect Dis 2002, 35:572-77.

11. Dymock D, Weightman AJ, Scully C, Wade WG: Molecular analysis of microflora associated with dentoalveolar abscesses. J Clin Microbiol 1996, 34:537-542.

12. Kroes I, Lepp PW, Relman DA: Bacterial diversity within the human subgingival crevice. Proc Natl Acad Sci USA 1999, 96:14547-14552.

13. Sakamoto M, Umeda M, Ishikawa I, Benno Y: Comparison of the oral bacterial flora in saliva from a healthy subject and two periodontitis patients by sequence analysis of $16 \mathrm{~S}$ rDNA libraries. Microbiol Immunol 2000, 44:643-652.

14. Paster BJ, Boches SK, Galvin JL, Ericson RE, Lau CN, Levanos VA, Sahasrabudhe A, Dewhirst FE: Bacterial diversity in human subgingival plaque. J Bacteriol 2001, 183:3770-3783.

15. Paster BJ, Falkler WA Jr, Enwonwu CO, Idigbe EO, Savage KO, Levanos VA, Tamer MA, Ericson RL, Lau CN, Dewhirst FE: Prevalent bacterial species and novel phylotypes in advanced noma lesions. J Clin Microbiol 2002, 40:2187-2191.

16. Aas JA, Paster BJ, Stokes LN, Olsen I, Dewhirst FE: Defining the normal bacterial flora of the oral cavity. J Clin Microbiol 2005, 43:5721-5732.

17. Becker MR, Paster BJ, Leys EJ, Moeschberger ML, Kenyon SG, Galvin JL, Boches SK, Dewhirst FE, Griffen AL: Molecular analysis of bacterial species associated with childhood caries. J Clin Microbiol 2002, 40:1001-1009.

18. Kumar PS, Griffen AL, Moeschberger ML, Leys EJ: Identification of candidate periodontal pathogens and beneficial species by quantitative 16 S clonal analysis. J Clin Microbiol 2005, 43:3944-3955.

19. Kumar PS, Leys EJ, Bryk JM, Martinez FJ, Moeschberger ML, Griffen AL: Changes in periodontal health status are associated with bacterial community shifts as assessed by quantitative $16 \mathrm{~S}$ cloning and sequencing. J Clin Microbiol 2006, 44:3665-3673.

20. Riep B, Edesi-Neuss L, Claessen F, Skarabis H, Ehmke B, Flemmig TF Bernimoulin JP, Gobel UB, Moter A: Are putative periodontal pathogens reliable diagnostic markers?. J Clin Microbiol 2009, 47:1705-1711.
21. Donlan RM, Costerton JW: Biofilms: survival mechanisms of clinically relevant microorganisms. Clin Microbiol Rev 2002, 15:167-193.

22. Cato EP, Moore LVH, Moore WEC: Fusobacterium alocis sp. nov. and Fusobacterium sulci sp. nov. from the human gingival sulcus. Int J Syst Bacteriol 1985, 35:475-477.

23. Jalava J, Eerola E: Phylogenetic analysis of Fusobacterium alocis and Fusobacterium sulci based on 16S rRNA gene sequences: proposal of Filifactor alocis (Cato, Moore and Moore) comb. nov. and Eubacterium sulci (Cato, Moore and Moore) comb. nov. Int J Syst Bacteriol 1999, 49(Pt 4):1375-1379.

24. Maiden MF, Tanner A, Macuch PJ: Rapid characterization of periodontal bacterial isolates by using fluorogenic substrate tests. J Clin Microbiol 1996, 34:376-384.

25. Ohta K, Makinen KK, Loesche WJ: Purification and characterization of an enzyme produced by Treponema denticola capable of hydrolyzing synthetic trypsin substrates. Infect Immun 1986, 53:213-220.

26. Loesche WJ: The identification of bacteria associated with periodontal disease and dental caries by enzymatic methods. Oral Microbiol Immunol 1986, 1:65-72.

27. Kumar PS, Griffen AL, Barton JA, Paster BJ, Moeschberger ML, Leys EJ: New bacterial species associated with chronic periodontitis. J Dent Res 2003, 82:338-344.

28. Dahlen $G$, Leonhardt A: A new checkerboard panel for testing bacterial markers in periodontal disease. Oral Microbiol Immunol 2006, 21:6-11.

29. Hutter G, Schlagenhauf U, Valenza G, Horn M, Burgemeister S, Claus H, Vogel $U$ : Molecular analysis of bacteria in periodontitis: evaluation of clone libraries, novel phylotypes and putative pathogens. Microbiology 2003, 149:67-75.

30. Siqueira JF Jr, Rocas IN: Detection of Filifactor alocis in endodontic infections associated with different forms of periradicular diseases. Oral Microbiol Immunol 2003, 18:263-265

31. Wecke J, Kersten T, Madela K, Moter A, Gobel UB, Friedmann A, Bernimoulin J: A novel technique for monitoring the development of bacterial biofilms in human periodontal pockets. FEMS Microbiol Lett 2000, 191:95-101.

32. Maidak BL, Cole JR, Lilburn TG, Parker CT Jr, Saxman PR, Farris RJ, Garrity GM, Olsen GJ, Schmidt TM, Tiedje JM: The RDP-II (Ribosomal Database Project). Nucleic Acids Res 2001, 29:173-174.

33. Amann RI, Binder BJ, Olson RJ, Chisholm SW, Devereux R, Stahl DA: Combination of $16 \mathrm{~S}$ rRNA-targeted oligonucleotide probes with flow cytometry for analyzing mixed microbial populations. App/ Environ Microbiol 1990, 56:1919-1925.

34. Loy A, Horn M, Wagner M: probeBase: an online resource for rRNAtargeted oligonucleotide probes. Nucleic Acids Res 2003, 31:514-516.

35. Armitage GC: Development of a classification system for periodontal diseases and conditions. Northwest Dent 2000, 79:31-35.

36. Syed SA, Loesche WJ: Survival of human dental plaque flora in various transport media. Appl Microbiol 1972, 24:638-644.

37. Moter A, Hoenig C, Choi BK, Riep B, Gobel UB: Molecular epidemiology of oral treponemes associated with periodontal disease. J Clin Microbiol 1998, 36:1399-1403.

38. Moter A, Leist G, Rudolph R, Schrank K, Choi BK, Wagner M, Gobel UB: Fluorescence in situ hybridization shows spatial distribution of as yet uncultured treponemes in biopsies from digital dermatitis lesions. Microbiology 1998, 144(Pt 9):2459-2467.

39. Schlafer S, Nordhoff M, Wyss C, Strub S, Hubner J, Gescher DM, Petrich A, Gobel UB, Moter A: Involvement of Guggenheimella bovis in digital dermatitis lesions of dairy cows. Vet Microbiol 2008, 128:118-125.

40. Sunde PT, Olsen I, Gobel UB, Theegarten D, Winter S, Debelian GJ Tronstad L, Moter A: Fluorescence in situ hybridization (FISH) for direct visualization of bacteria in periapical lesions of asymptomatic root-filled teeth. Microbiology 2003, 149:1095-1102.

41. Daims $H$, Lucker $S$, Wagner $M$ : daime, a novel image analysis program for microbial ecology and biofilm research. Environ Microbio/ 2006, 8:200-213.

42. ten Cate JM: Biofilms, a new approach to the microbiology of dental plaque. Odontology 2006, 94:1-9.

43. Listgarten MA: Structure of the microbial flora associated with periodontal health and disease in man. A light and electron microscopic 
study. J Periodontol 1976, 47:1-18.

44. Marchesi JR, Sato T, Weightman AJ, Martin TA, Fry JC, Hiom SJ, Dymock D, Wade WG: Design and evaluation of useful bacterium-specific PCR primers that amplify genes coding for bacterial 16S rRNA. Appl Environ Microbiol 1998, 64:795-799.

45. Rickard AH, Gilbert P, High NJ, Kolenbrander PE, Handley PS: Bacterial coaggregation: an integral process in the development of multi-species biofilms. Trends Microbiol 2003, 11:94-100.

doi:10.1186/1471-2180-10-66

Cite this article as: Schlafer et al:: Filifactor alocis - involvement in periodontal biofilms. BMC Microbiology 2010 10:66.

Submit your next manuscript to BioMed Central and take full advantage of:

- Convenient online submission

- Thorough peer review

- No space constraints or color figure charges

- Immediate publication on acceptance

- Inclusion in PubMed, CAS, Scopus and Google Scholar

- Research which is freely available for redistribution

Submit your manuscript at www.biomedcentral.com/submit 